\title{
SPECTROSCOPIC OBSERVATIONS OF U GEM
}

\author{
J. I. SM A K
}

Institute of Astronomy, Polish Academy of Sciences, Warsaw, Poland

\begin{abstract}
Radial velocities of U Gem have been measured from individual components of the emission lines of the Balmer series, Ca II K, and He I 4471, originating in the gaseous disk and in the hot spot of this binary system. These new data give $K_{1} \cong 143 \mathrm{~km} \mathrm{~s}^{-1}$ and $V_{\text {disk }} \sin i \cong 530 \mathrm{~km} \mathrm{~s}^{-1}$. The resulting mass of the primary component is $m_{1}=1.1 \pm 0.4 m_{\odot}$ and the mass-ratio $m_{2} / m_{1}=0.6 \pm 0.2$. Radial velocities and intensities of the emission components originating in the hot spot are also discussed.
\end{abstract}

\section{Radial Velocity Data}

This is a preliminary report on the results of the radial velocity measurements based on a new series of over 60 spectrograms, at a dispersion of $90 \AA \mathrm{mm}^{-1}$, obtained in JanuaryFebruary 1974 with the Cassegrain CIT spectrograph of the 84 -in. $(213-\mathrm{cm})$ reflector of the Kitt Peak National Observatory. An attempt was made to measure on these plates, whenever possible, the two components of the emission lines originating in the rotating gaseous disk and the third component due to the hot spot. For discussion of the relevant observational data and of a model of U Gem see Kraft (1962), Krzeminski (1965), Smak (1971) and Warner and Nather (1971).

Figure 1 shows, as an example, the radial velocity data based on the $\mathrm{H} \delta$ line. The two sets of points correspond to the red and blue components originating in the disk; the open circles indicate cases when either of these components in blended with the third component due to the spot, or cases when the emission line profile was affected by the eclipse. When the red or blue component is not contaminated by any such effects, we can write:

$$
V_{r, b}=\gamma \pm V_{\mathrm{disk}} \sin i+K_{1} \sin \left(\varphi+\varphi_{0}\right)
$$

where $V_{\mathrm{disk}} \sin i$ is the projected rotational velocity of the outer parts of the disk (Smak, 1969 ) and $K_{1}$ is the projected orbital velocity of the primary component. A least squares solution of this equation based on all lines measured gives

$$
V_{\mathrm{disk}} \sin i=530 \pm 7 \mathrm{~km} \mathrm{~s}^{-1}, \quad \mathrm{~K}_{1}=143 \pm 10 \mathrm{~km} \mathrm{~s}^{-1} \text {. }
$$

The relatively small formal errors of this determination should, however, be treated with caution because the solutions based on individual lines show a considerable scatter. For example, the $H \delta$ solution, as shown in Figure 1 , gives $V_{\text {disk }} \sin i=544 \pm 17$, and $K_{1}$ $=178 \pm 14 \mathrm{~km} \mathrm{~s}^{-1}$.

\section{The Masses of the Components}

The two parameters supplied by observations, namely $V_{\text {disk }} \sin i$ and $K_{1}$, are directly related to the masses of the components. For our purposes the two relations can be written in the form

$$
m_{1}=(2 \pi G)^{-1} K_{1}^{3}\left(1+\frac{m_{1}}{m_{2}}\right)^{2} \frac{m_{1}}{m_{2}} P \operatorname{cosec}^{3} i,
$$




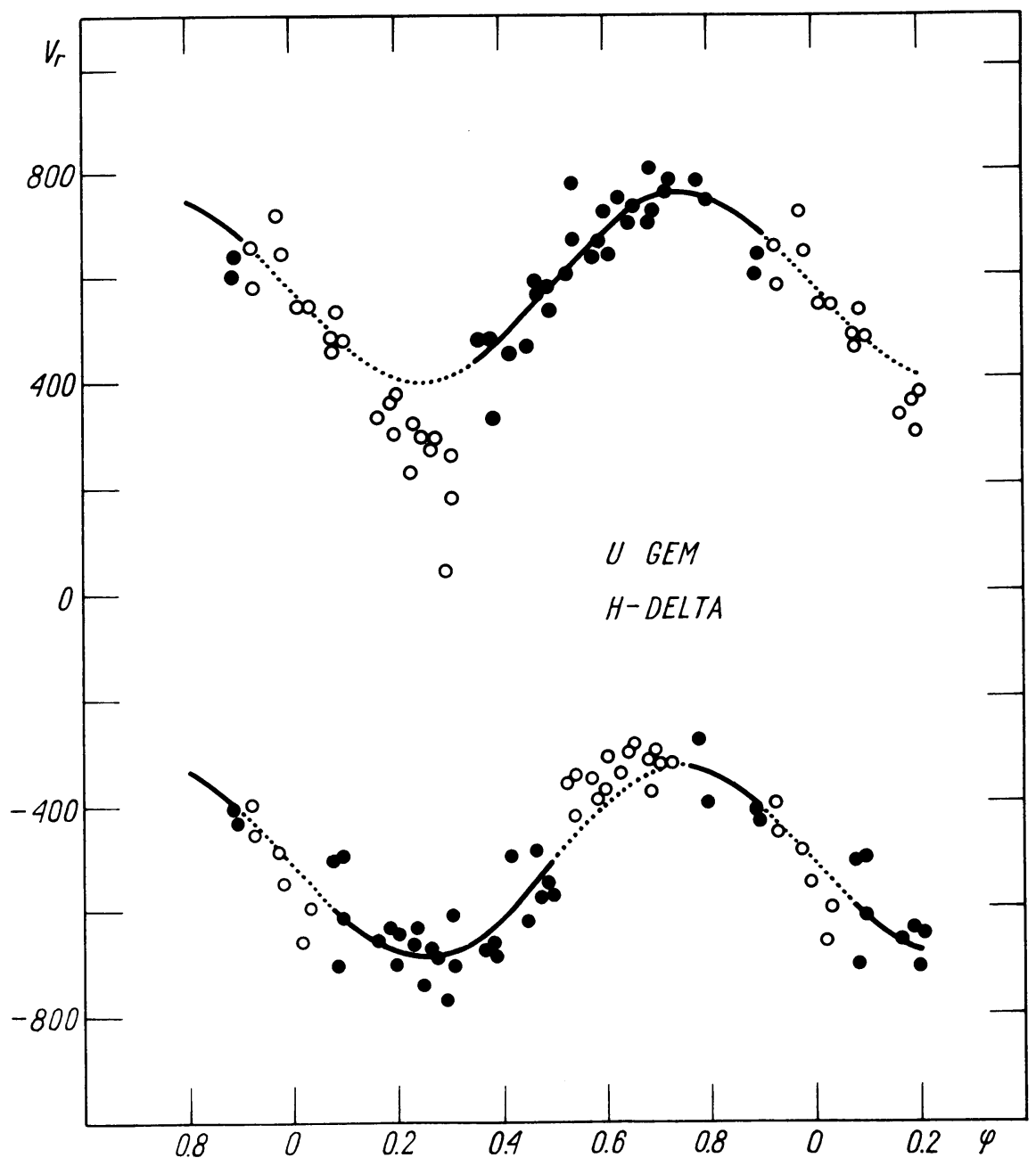

Fig. 1. Radial velocities of U Gem from the H $\delta$ line. Filled circles (and solid lines) indicate observations (and phase intervals) used for the solution given in the text. See text for other details.

$$
m_{1}=(2 \pi G)^{-1}\left(V_{\text {disk }} \sin i\right)^{3}\left(1+\frac{m_{1}}{m_{2}}\right)^{1 / 2} r_{\text {disk }}{ }^{3 / 2} P \operatorname{cosec}^{3} i,
$$

where $r_{\text {disk }}$ is the outer radius of the disk in units of the separation (other parameters are in cgs units), and can be approximately identified with the distance of the hot spot from the central star which, for U Gem, was estimated at 0.35 (Smak, 1971). Each of these two equations permits one to determine $m_{1}$ as a function of the mass ratio. The two of them combined permit one to determine $m_{1}$ as well as the mass ratio. The situation is illustrated in Figure 2 which shows two pairs of curves with $V_{\text {disk }} \sin i=500$ and $550 \mathrm{~km} \mathrm{~s}^{-1}$ and $K_{1}=150$ and $180 \mathrm{~km} \mathrm{~s}^{-1}$. With $V_{\text {disk }} \sin i=530$ and $K_{1}=143 \mathrm{~km} \mathrm{~s}^{-1}$, and with crude estimates of the uncertainties involved we get

$$
m_{1}=1.1 \pm 0.4 m_{\odot} \text { and } m_{2} / m_{1}=0.6 \pm 0.2 \text {. }
$$




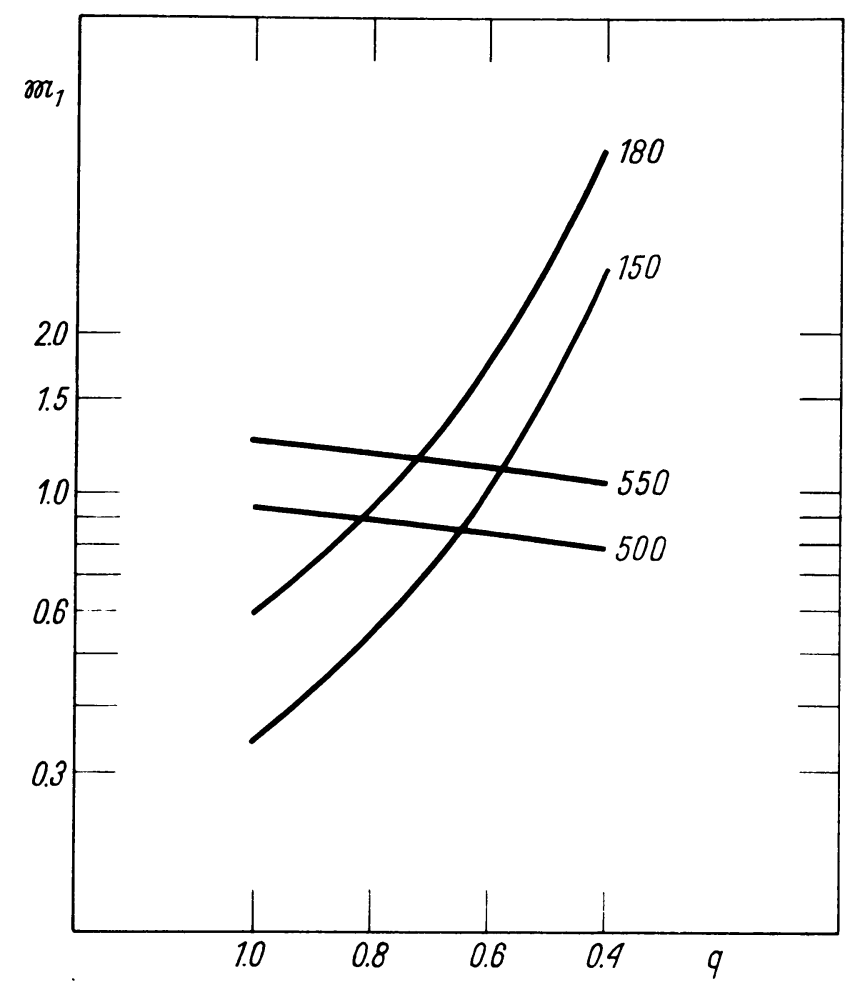

Fig. 2. The mass and mass-ratio determination from the two relations discussed in the text. Two pairs of curves are shown for illustrative purposes.

The most serious uncertainty is connected with the assumption of the so-called Keplerian motion of the outer parts of the disk, incorporated into the relation including $V_{\text {disk }} \sin$ $i$, and this is rather difficult to evaluate. Our solution gives also the mass and radius of the secondary component and the orbital radius:

$$
\begin{aligned}
& m_{2}=0.6_{5} \pm 0.4 m_{\odot}, \quad R_{2}=0.5 \pm 0.1_{5} R_{\odot}, \\
& A=1.1 \pm 0.2 \times 10^{11} \mathrm{~cm} .
\end{aligned}
$$

It can be seen that the mass and radius of the secondary component are - within errors in agreement with the mass-radius relation for lower main sequence stars.

\section{The Hot Spot}

The hot-spot component of the emission lines of the Balmer series and Ca II K can be clearly seen on nearly all plates and - at certain phases - it can be measured as a separate feature, in addition to the two components originating in the disk. The He I 4471 line, which is weakly present (but not always measurable) on all plates, appears to originate exclusively in the hot spot. The results of radial velocity measurements of the hot-spot component is presented in Figure 3. The helium-line results show less scatter and appear to define a fairly regular sine-wave curve: 


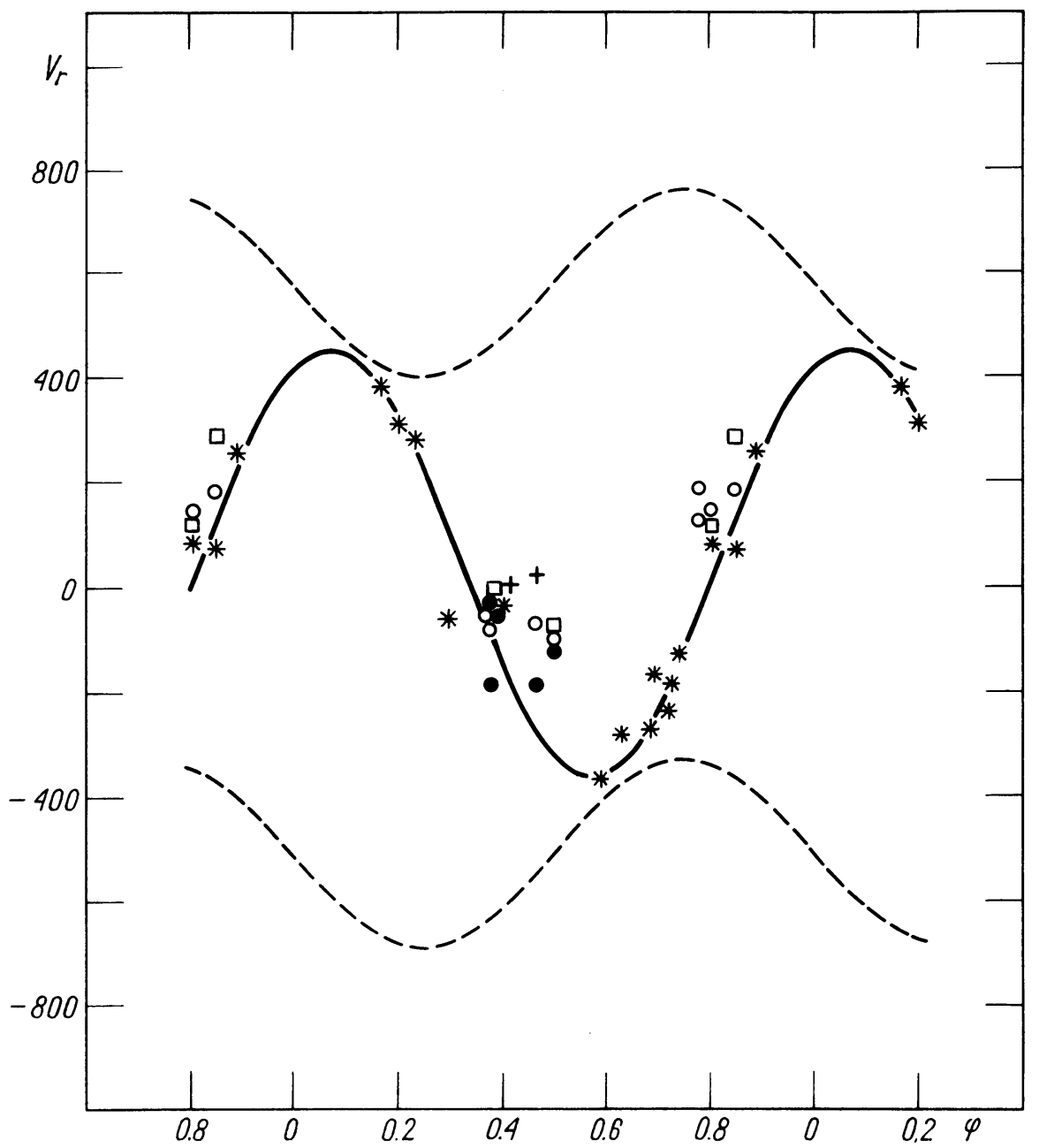

Fig. 3. Radial velocities from the hot-spot components. Asterisks are measurements based on the He I 4471 line. Other symbols correspond to the Balmer lines and Ca II K. The solid line represents the solution based on He I 4471, as discussed in the text. The broken lines are the curves from Figure 1.

$$
V_{\text {spot }}=\gamma+A \sin 2 \pi\left(\varphi+\varphi_{0}\right)
$$

The least squares solution gives:

$$
A=404 \pm 39 \mathrm{~km} \mathrm{~s}^{-1}, \quad \varphi_{0}=0.181 \pm 0.013 \text {. }
$$

Using these numbers and the value of $m_{2} / m_{1}$ obtained earlier we can find that the velocity of radiating atoms in the system of coordinates co-rotating with the binary components amounts only to about $390 \mathrm{~km} \mathrm{~s}^{-1}$ and that the velocity vector forms with the line joining the two components an angle of only about $30^{\circ}$. Such a direction is certainly closer to the direction of the impinging stream than to the direction implied by the rotation of the outer parts of the disk. This seems to be a very important piece of information 
pertaining to the model of the hot spot.

As a result of blending of the hot-spot component with the two components originating in the disk, the emission line spectrum of $U$ Gem displays strong $V / R$ variations. These are illustrated in Figure 4, where visual estimates (a more detailed spectrophotometry is planned) of $V / R$ are plotted against phase for all plates published by Kraft (1962) and all plates of the present series. While both plots clearly show the $V / R$ variations, their intercomparison reveals the following surprising difference. In 1961/62 the $V$-component, when observed as the stronger one at $\varphi \approx 0.7$, was considerably stronger than the $R$-component; the $R$-component, when observed as the stronger one at $\varphi \approx 0.2$, was only slightly stronger than the $V$-component. And this was, in fact, consistent with the model of UGem, predicting the best visibility of the spot at phases shortly before the eclipse (Smak, 1971). The picture obtained in 1974, however, shows an opposite effect, namely that the best visibility of the hot-spot component is at $\varphi \approx 0.2$. Whatever the explanation

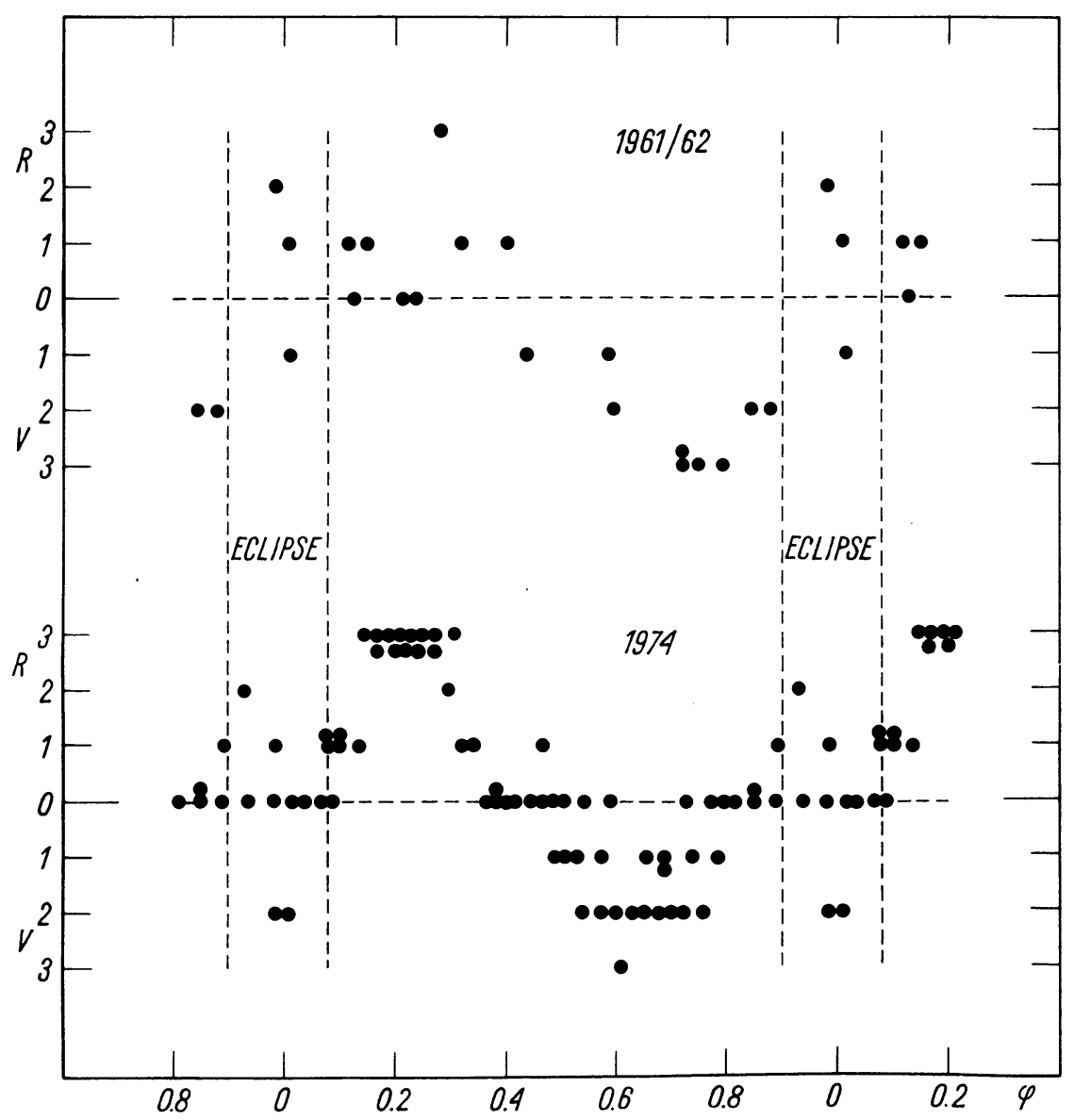

Fig. 4. The $V / R$ variations in the spectrum of $U$ Gem in 1961/62 (Kraft, 1962) and in 1974 (present study). Estimates are on an arbitrary scale, with $R=3$ meaning the red component much stronger than the violet one, and $V=1$ meaning the violet component slightly stronger than the red one, etc. 
of this effect, it appears quite obvious that the line radiation must come from a somewhat different area than the continuous radiation of the hot spot and/or that the absorption effects in the outer parts of the disk are quite different for the line radiation and for the continuum radiation. Again, this seems to be an important piece of information concerning the structure of the hot spot.

This investigation is based on material collected at the Kitt Peak National Observatory in 1974, at the time the author held a visiting professorship at the Astronomy Department of the Ohio State University.

\section{References}

Kraft, R. P.: 1962, Astrophys. J. 135, 408.

Krzemiński, W.: 1965, Astrophys. J. 142, 1051.

Smak, J.: 1969, Acta Astron. 19, 155.

Smak, J.: 1971, Acta Astron. 21, 15.

Warner, B. and Nather, R. E.: 1971, Monthly Notices Roy. Astron. Soc. 152, 219.

\section{DISCUSSION}

Eggleton: If radiation associated with hot spot is actually coming from further upstream, can this affect derived parameters like mass ratio and primary mass?

Smak: It can, because they depend on the assumption $V_{\text {disk }}=V_{\text {spot }}$. I believe, however, that the uncertainties should not be too drastic and I hope that my estimated errors of $M_{1}$ and $M_{2} / M_{1}$ cover that. 